\title{
LAB-ON-A-CHIP WITH FLUID ACOUSTIC MICROAGITATION Piezoelectric Polymer $\beta$-PVDF used as Ultrassonic Transducer
}

\author{
V. F. Cardoso, J. G. Rocha, F. O. Soares, G. Minas \\ Dept. Industrial Electronics, University of Minho, Campus de Azurem, 4800-058 Guimaraes, Portugal \\ vanessa@dei.uminho.pt,gerardo@dei.uminho.pt,filomena.soares@dei.uminho.pt,gminas@dei.uminho.pt \\ S. Lanceros-Mendez \\ Dept. Physics, University of Minho, Campus de Gualtar, 4710-057 Braga, Portugal \\ lanceros@fisica.uminho.pt
}

Keywords: Lab-on-a-chip, PVDF, acoustic microagitation, SU-8.

Abstract: The main objective of this article is to describe the development of a fully-integrated disposable lab-on-achip for point of care testing and monitoring of biochemical parameters in biological fluids. The lab-on-achip is composed mainly by two dies: the fluid and the detection. The fluid die, fabricated in SU-8, comprises three microfluidic cuvettes, containing the fluids into analysis, and a $\beta$-PVDF microagitation system. The detection die is fabricated in a CMOS standard process and contains the photodetectors and the electronics for signal actuation and detection. The main innovation of this lab-on-a-chip is the application of an acoustic microagitation technique by the deposition of the $\beta$-PVDF piezoelectric polymer underneath the microfluidic structures, with automatic electronic control. This piezoelectric polymer produces mechanical vibrations, which allow the enhancement of the mixing and the reaction.

\section{INTRODUCTION}

Microfluidic technology has become an important tool for analytical biochemistry applications. It enables the fabrication of precise and small structures in glass, quartz, silicon or polymeric materials: the lab-on-a-chip concept. The great interest in that technology stems from the inherent performance gains: reduced sample size, higher degree of integration and thus enhanced potential for automation and control fluids of submicroliter volumes, shortened response time, potential for improved analytical performance, reduced storage of chemicals and hence laboratory safety, and reduced costs (Kopp et. al, 1997).

There is a large demand in the healthcare system to develop lab-on-a-chips for rapid and reliable point of care (POC) testing and monitoring. Such lab-ona-chips would significantly enhance the quality of a diagnostic by offering immediate measurement of several clinically relevant parameters that can be used to assess the health of the patient. The biochemical analysis of the patient's biological fluids is a good start. Most diseases leave a molecular fingerprint in those fluids and by measuring that fingerprint in the right way, the precision of the diagnostic can be improved (Connolly, 1995). However, the physician or the patient has no routine in performing the advanced biochemical analysis on-chip. Therefore, to develop point of care disposable lab-on-a-chips, avoiding cross-contamination of samples and measurements errors, it is necessary to have a reliable and highly automated microfluidic control system. This system should be fully integrated with the control and the detection electronics implemented on a low-cost substrate and performed by a low-cost fabrication process. The use of MEMS (Micro Electro Mechanical Systems) based devices, such as microvalves and micropumps, increases the cost of the system, needs complex control systems and are difficult to integrate (Reyes et. al, 2002). Mixing only by diffusion avoids these drawbacks. However, long transit times and consequently long microchannels are necessary, if large molecules with small diffusivities must react. This illustrates how dramatic the diffusion limitation is.

To overcome the long transit times due to diffusion and to enable high-efficient reactions it is 
necessary to induce the microfluidic die by a mechanism that accelerates the mixing and the reaction, preferably with no moving parts. In this paper, such mechanism is presented.

\section{BACKGROUND}

The need for rapid and in-situ measurements with low sample volumes has led to the development of miniaturized analyses devices with the fluidic, detection and readout systems integrated in a single chip. The vision of those devices was presented in 1990 (Manz et. al., 1990) and has been since then the inspiration for an intense research effort pursuing miniaturization of macroscopic biochemical analyses methods. Within the field of clinical diagnostics, several lab-on-a-chip approaches have been published (Auroux et. al, 2002, Reyes et. al., 2002).

Our group has developed a lab-on-a-chip for measuring the concentration of some biomolecules in urine samples by optical absorption technique (Minas, et. al., 2005). It comprises three parts in a multi-chip-module: (1) a microfluidic system die containing the microchannels fabricated using SU-8 techniques (Ribeiro et. al., 2005); (2) an optical filtering system based on highly selective FabryPerot optical resonators using a stack of CMOS process compatible thin-film layers (Minas et. al., 2006); (3) a detection and readout system fabricated in a CMOS microelectronic process (Minas et. al., 2004). The optical filtering system enables the measurement using white light illumination, thus avoiding the use of a wavelength dependent light source. This characteristic makes the lab-on-a-chip portable and ensures that the analysis can be performed at any location with instantaneous results. However, in that lab-on-a-chip, mixing the samples with the reagents was performed by diffusion, which leads to long transit times, especially when large molecules with small diffusion coefficients must be analysed. Therefore, to be valuable for point of care testing and monitoring, the microfluidic die of the lab-on-a-chip requires a microagitation mechanism. It is desirable that this mechanism does not require any external apparatus, internal moving parts or valves.

The flows used in microfluidic systems are very small, of the order of $100 \mu \mathrm{m}$, with velocities of the order of $1 \mathrm{~mm} / \mathrm{s}$, which leads to Reynolds number less than 1 . For this small Reynolds numbers the flow is dominated by the viscous effects and turbulent mixing is impossible. Therefore, mixing in a straight channel rely on diffusion, but since diffusion coefficients of some biological species (e.g. enzymes and other proteins) are very small, mixing may take several minutes or even hours. This may be undesirable for some applications and alternative mixing methods must be developed.

Acoustic waves are an interesting solution for this problem. They have been used both to promote mixing (Bengtsson et. al., 2004) and to pump fluids (Rife et. al., 2000). Sound waves that propagate in the fluid generate pressure differences that induce the so called acoustic streaming. Secondary flows can be created in the plane perpendicular to the main flow in the channel. These secondary flows promote mixing by convection between otherwise parallel currents. Therefore, acoustic microagitation could be a simple actuation source for mixing and promoting fluids reaction.

In order to produce the ultrasounds, the channels and the reaction chambers of the microfluidic die must have an ultrasound transducer, which can be fabricated from a piezoelectric polymer, such as the PVDF - Poly(Vinylidene Fluoride) in its beta-phase. $\mathrm{PVDF}$ is a polymer with interesting piezoelectric properties, which allows important electro-optical, electromechanical and biomedical applications. This polymer shows at least four crystalline phases. The one with the best piezo- and pyroelectric properties, after poling, is the beta-phase (Sessler, 1987). Until recently, this phase was exclusively obtained by mechanical stretching of films originally in the nonpolar alfa-phase, which is the most stable one from a thermodynamic point of view and directly obtained from the melt. This process results in films mostly in the beta-phase, but with a small percentage of alfaphase material (Sencadas et. al., 2004). Unoriented films exclusively in the beta-phase were obtained from the crystallization of PVDF from solution with N,N-Dimethyl Formamide or Dimethyl Acetamide at temperatures below $70^{\circ} \mathrm{C}$. The electromechanical properties of this film were improved by a patented process by our group (Lanceros-Mendez et. al., 2006).

\section{DEVICE DESCRIPTION}

This paper describes the concept, operation and implementation of a portable, low-cost, plastic based, disposable and highly automated lab-on-achip for biochemical analyses of biological fluids. It uses optical absorption spectrophotometry as measurement analytical technique and acoustic microagitation for mixing the fluids. Its application is for point-of-care systems. 


\subsection{Measurement Analytical Technique}

The spectrophotometric analysis, the study of the interaction of electromagnetic radiation with (bio)chemical compounds, is a very convenient and often used analytical technique in clinical laboratories for routine tests analyses, especially the ones based on colorimetric detection (Sigma, 2006). However, many of the analytes of interest for clinical analysis do not have chromophores that absorb light in a useful part of the visible range. Specific chemical reagents are available to transform these analytes into colored products that do have adequate absorbance. Therefore, in that detection method, the sample (ex: urine) is mixed with a proper reagent for the biochemical parameter in analysis (ex: uric acid reagent) and from that mixture, a visible colour is produced. The intensity of that colour is proportional to the biochemical parameter concentration and can be quantified by measuring the optical absorption of the mixture, once the mixture has an absorption maximum at a specific wavelength. As an example, for determining the uric acid concentration in a urine sample, the mixture has an absorption maximum at $495 \mathrm{~nm}$ (Figure 1). For an accurate measurement a complete and homogeneous mixing must be achieved.

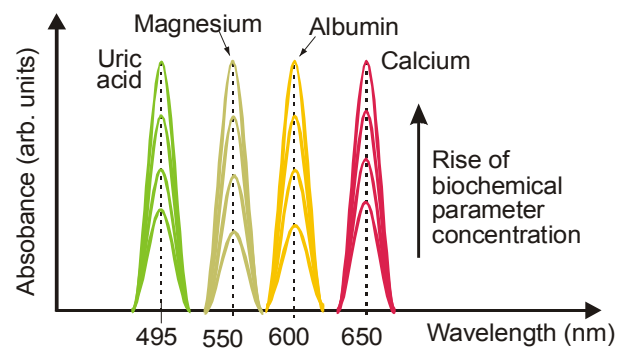

Figure 1: Absorption spectra for some biochemical parameters with different concentrations in urine.

\subsection{Lab-on-a-chip Operation}

The lab-on-a-chip is composed by two dies: the fluidic die and the detection die (Figure 2). For the measurement of the concentration of the biochemical parameters, a light beam is directed to the three fluidic cuvettes. The intensity of the light beam transmitted through the mixture is measured using the three photodetectors, placed underneath the microfluidic cuvettes, forming three optical channels. The photodetectors signal is converted, by the readout electronics, into a digital signal that allows simpler computer interfacing. The accurate sample and reagent mixing is performed by the control electronics that actuates an electroactive polymer $(\beta$-PVDF), deposited underneath the microfluidic cuvettes. This polymer vibrates and produces the proper acoustic microagitation of the mixture, e. g., the produced waves are coupled to the slide and propagate into the microfluidic cuvette.

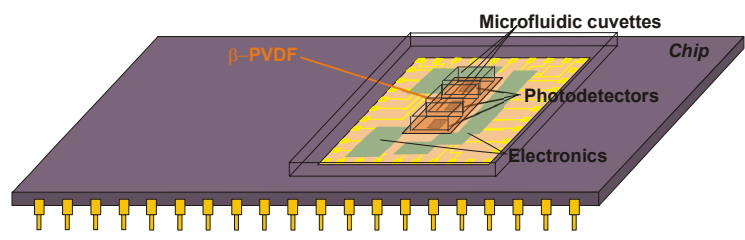

Figure 2: Schematic drawing of the lab-on-a-chip structure.

\subsection{Microfluidic Die}

The microfluidic die includes the microfluidic cuvettes containing the fluids to be analysed (Figure 2). Three cuvettes are needed for each analysis: one for the chemical reagent, in order to obtain the baseline reference; other for the mixture of the sample plus the reagent, to perform the analysis of the coloured mixed solution; and a third one with a standard sample with a well-known concentration of the biochemical parameter that is being analyzed, for the calibration of the biochemical parameter concentration.

Under the microfluidic cuvettes a piezoelectric polymer, $\beta$-PVDF, is deposited (Figure 2). This material will be responsible for the acoustic microagitation of the fluid. $\beta$-PVDF is a transparent piezoelectric polymer that will produce the necessary vibration, when an electrical alternating voltage is applied to its contacts. These vibrations result in the acoustic microagitation necessary to mix the sample with the reagent inside the cuvettes. Moreover, it accelerates the required time for the complete and homogeneous mixing, improving the efficiency of the reaction.

The microfluidic cuvettes are fabricated using a photoplastic material: the photoresist SU-8. This photoresist is an epoxy-based material that offers relevant properties, such as high mechanical strength, good adhesion on many different substrate materials and biocompatibility. The SU- 8 based fabrication is a low-cost process, UV (from $350 \mathrm{~nm}$ to $400 \mathrm{~nm}$ ) lithography semiconductor compatible and does not require expensive masks. It can be processed by using a spincoating and an UV maskaligner. In addition, the microfluidic system can be a disposable die, which minimize the costs associated with cleaning of the microfluidic cuvettes and avoids the contamination between analyses. 
Moreover, SU-8 based processing enables the fabrication of deep structures with very low sidewall roughness which is suitable for optical absorption measurements (IBM, 1989).

The negative mask to be used for patterning the structure of the microfluidic cuvettes is fabricated from a regular transparency foil, such as the ones used in printed circuit boards. The chosen SU-8 photoresist is the SU-8-2150, which has a high viscosity and is the most appropriate for the required depth $(\geq 500 \mu \mathrm{m})$. The following paragraphs describe the microfluidic cuvettes processing steps, including the SU-8 and the $\beta$-PVDF depositions.

After cleaning and drying the glass substrate, a thin-film of Itrium-Tin-Oxide (ITO) of approximately $40 \mathrm{~nm}$ is deposited by PVD, in order to form the bottom contact of the piezoelectric $\beta$-PVDF. Then, a $600 \mathrm{~nm} \beta$-PVDF layer is spun of on top of the ITO. The coated $\beta$-PVDF is activated by applying an electrical field of tens of megavolts by meter, which is known as the electrical poling of the polymeric material. After that, the top electrical contact of the $\beta$-PVDF is deposited using the same procedure used for the bottom contact. These steps complete the fabrication of the microagitation mechanism.

Next, the fabrication of the microfluidic cuvettes starts with the spin of $1.25 \mathrm{ml} / \mathrm{cm}^{2}$ of SU-8 on top of the $\beta$-PVDF top ITO contact. This process requires two steps: first, ramping up the spin rotation to $500 \mathrm{rpm}$ during $5 \mathrm{~s}$ and keeping that velocity for $10 \mathrm{~s}$; second, ramping up again the spin rotation up to $1000 \mathrm{rpm}$ during $3 \mathrm{~s}$ and keeping it for $30 \mathrm{~s}$. Then, a soft bake process is needed for evaporation of the solvent and for the hardening of the SU-8. The soft bake is performed at $65^{\circ} \mathrm{C}$ during $420 \mathrm{~s}$ and at $95{ }^{\circ} \mathrm{C}$ during $3600 \mathrm{~s}$. The initial temperature allows a slower evaporation, which results in a better coating and, mainly and very important, without grooves. Then, the cuvettes are exposed to UV light, using a negative mask, for patterning the structure. Figure $3 \mathrm{a}$ shows a cross-section of the device after the aforementioned steps.

An excessive dose of light with wavelength lower than $350 \mathrm{~nm}$ results in an excessive absorption at the surface of the film. The effect can be a pyramidal shape of the microfluidic cuvettes. To avoid this effect, a commercially available glass optical filter was used to cut of the wavelengths lower than $350 \mathrm{~nm}$. The used exposition energy was $700 \mathrm{~mJ} / \mathrm{cm}^{2}$. The post-exposure bake is performed at $65^{\circ} \mathrm{C}$ during $60 \mathrm{~s}$, followed by $900 \mathrm{~s}$ treatment at $95^{\circ} \mathrm{C}$. The fabrication ends with the development, during 2400 s, with the SU-8 developer: an agitation bath of 1 methoxy-2-propanol acetate. In this way, the unexposed resist is dissolved and the microfluidic cuvettes are formed. The structure is then cleaned with isopropanol and deionised water and dried with nitrogen. Figure $3 b$ shows a cross section of the patterned structure. The structure of the microfluidic cuvettes is shown in Figure 4. Each cuvette is $1 \mathrm{~mm}$ wide, long and height, having a $1 \mu \mathrm{l}$ sample volume.

(a)

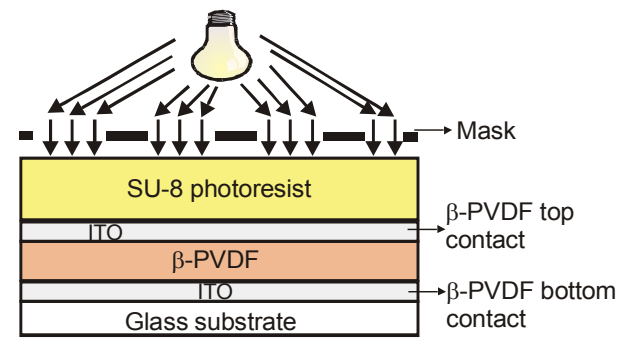

(b)

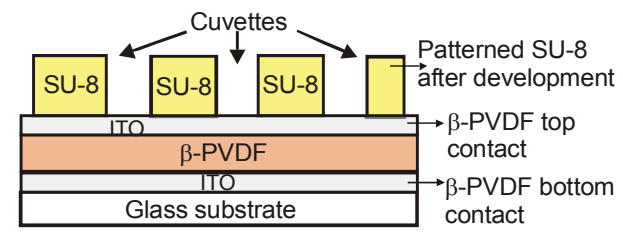

Figure 3: Fabrication sequence of the microfluidic cuvettes of the prototype: (a) deposition, spin coating, soft bake, UV exposure; (b) development of the SU-8.

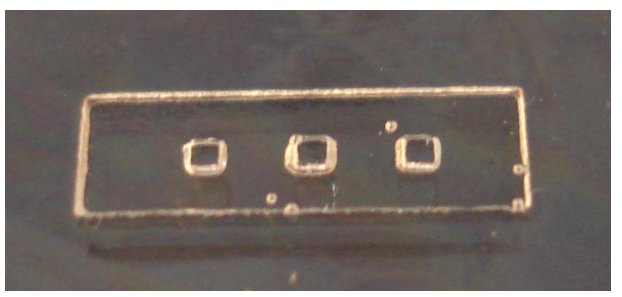

Figure 4: Picture of the microfluidic cuvettes.

\subsection{Detection Die}

The detection die includes the detectors and the electronics for signal actuation and detection, all fabricated in CMOS technology. Specifically, it comprises the photodetectors, its readout electronics and the electronics that control the voltages and frequencies applied to the $\beta$-PVDF. Figure 5 shows a block diagram of the photodiode and its readout electronics.

The readout electronics consists basically in a current amplifier and in a sigma-delta analog to digital converter. The circuit is repeated for each microfluidic cuvette. An additional circuit for the photodiode dark current compensation is also implemented. 
After the light reaches the photodiodes, the four analog to digital converters start the conversion and their output signal is placed in four separated lines. Further computer processing perform additional calculations of these four output signals to achieve the concentrations of the biochemical parameters into analysis. The oversample frequency of the sigma-delta converters is determined by the desired number of output bits (signal to noise ratio). For this particular application, an oversampling ratio of 64 and a first order one-bit sigma-delta analog to digital converter is adequate since the input signal has no time variations, allowing a high oversample ratio without the need for a high clock frequency.

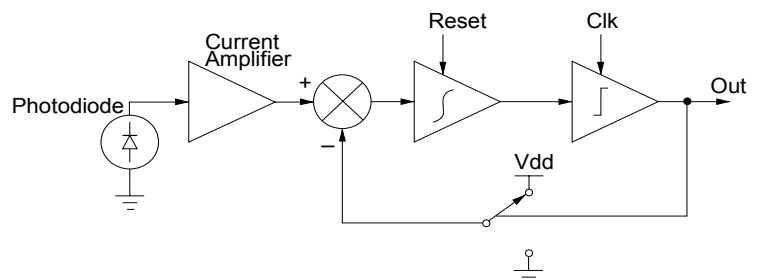

Figure 5: Block diagram of the readout electronics for each microfluidic cuvette.

For these sigma-delta converters, the gain of the integrator is very high, so, it is finite and larger than the oversampling ratio. At these conditions, the noise in the signal band increases only $0.15 \mathrm{~dB}$ (Candy et. al., 1992). Finally, the integrator must be initialized with a known voltage level at the beginning of each conversion. This initialization allows an improvement in the sigma-delta modulator of $3 \mathrm{~dB}$ in the signal to noise ratio (Netravali, 1977).

It was used a digital filter with constant weights (simple average) and the Pearson coefficient obtained for the response curve is larger than 0.99, which demonstrates a good linearity of the device. This result can even be improved by increasing the oversampling ratio and by using an optimized digital filter. Figure 6 shows a picture of the detection die.

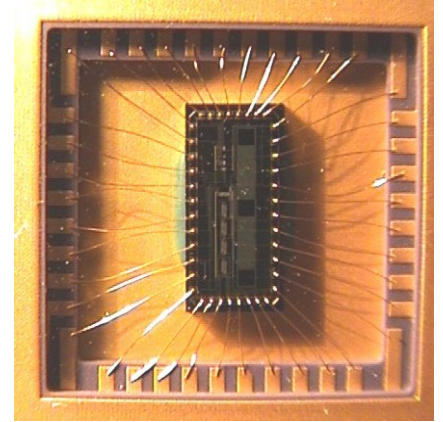

Figure 6: Picture of the detection die.

\section{EXPERIMENTAL RESULTS}

The evaluation of the mixing process was carried out using the Sigma Diagnostic kit (Infinity ${ }^{\mathrm{TM}}$ Uric Acid Reagent) and standards of urine with $30 \mathrm{mg} / \mathrm{dl}$ of uric acid concentration (Sigma, 2006). The reagent reacts with a sample of urine containing uric acid in a 50:1 ratio, and produces a pink colour with an absorption maximum at $495 \mathrm{~nm}$. Its manual procedure states that a gentle inversion of the cuvette that contains the mixture is enough for the complete and homogeneous mixing. Performing this manual agitation, and after approximately 5 minutes at room temperature, the mixing is complete. Without any agitation and due to the high diffusion coefficients of uric acid in the Infinity ${ }^{\mathrm{TM}}$ reagent, the complete mixing is achieved but it takes approximately 15 minutes at room temperature. In clinical laboratories, the macroscopic equipments have mechanical agitation of the cuvettes for improving mixing and reducing the reaction time.

The experimental results for evaluating the mixing process are presented in Figure 7. The microagitation was performed by powering the $\beta$-PVDF though the electrical contacts, with a sinusoidal signal of $5 \mathrm{~V}$ amplitude at the frequencies shown in the Figure 7. The system was calibrated for an absorbance of $0 \mathrm{a}$. u. filling the cuvette with deionised water, and for an absorbance of $1 \mathrm{a}$. u. filling the cuvette with the perfectly mixed solution (perfectly mixing was guaranteed by external agitation of the mixture using a slow-rotation vortex).
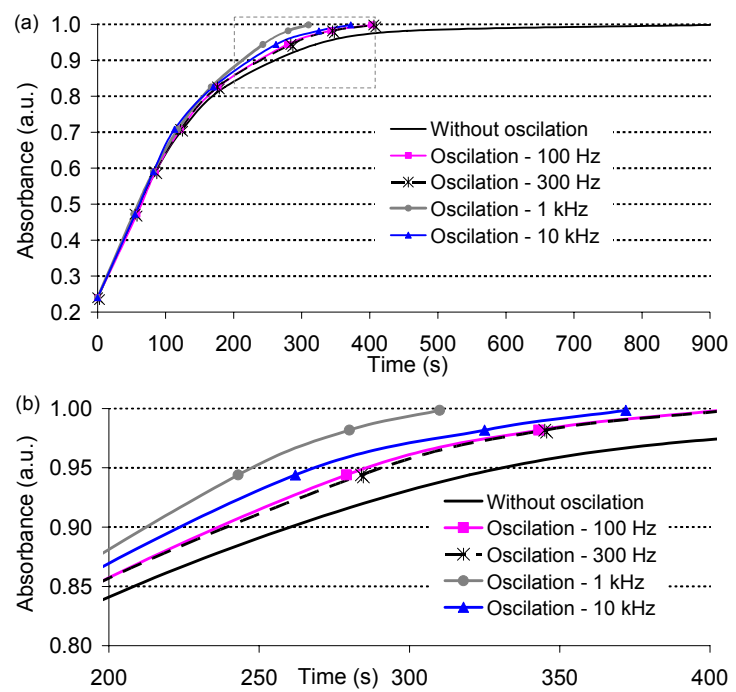

Figure 7: (a) Measured absorbance at $495 \mathrm{~nm}$ for $30 \mathrm{mg} / \mathrm{dl}$ of uric acid concentration in urine as a function of time for different driving frequencies. (b) Zoom of dashed-square. 
The analysis cuvette is filled with the reagent. Its measured absorbance is 0.24 a. u.. Afterwards, the urine standard with $30 \mathrm{mg} / \mathrm{dl}$ of uric acid is dispensed on the reagent. The absorbance increases as the sample is being mixed with the reagent. From Figure 7 it can be seen that with the application of oscillations, the mixing occurs in a faster way, being the time necessary to obtain the complete mixing (absorbance of $1 \mathrm{a}$. u.) at $1 \mathrm{kHz}$ only one third $(300 \mathrm{~s})$ of the complete mixing time without oscillation (900 s).

From those results it can be concluded that the application of acoustic microagitation by the $\beta$-PVDF piezoelectric polymer improves the mixing time. Moreover, uric acid has high diffusion coefficients, which is a good characteristic for mixing by diffusion only: after 15 minutes the mixture will be completed, even without agitation. However, when other biochemical parameters present in biological fluids have to be analysed, such as enzymes and some macromolecules, the mixing by diffusion can take hours or can even not occur. An example is a DNA analysis when PCR (Polymerase Chain Reaction) must be performed.

\section{CONCLUSIONS}

A lab-on-a-chip device with fluidic acoustic microagitation that reduces the mixing time of the analytes with the reagents was reported. The device consists of two dies: a microfluidic die, composed by three cuvettes and a $\beta$-PVDF acoustic microagitator, fabricated on a glass substrate; and an electronic detection die, composed by the readout circuits and the microagitation control electronics. The main innovative concept is the application of a $\beta$-PVDF film that produces acoustic microagitation, increasing the mixing velocity. Experimental results show that at $1 \mathrm{kHz}$ microagitation, the mixing time is reduced to one third of the time needed without microagitation. As a conclusion, it can be stated that, for decreasing device sizes, acoustic microagitation becomes a preferred technology for effective mixing.

\section{ACKNOWLEDGEMENTS}

Work supported by the Portuguese Science Foundation (grants PTDC/BIO/70017/2006 and POCI/CTM/59425/2004).

\section{REFERENCES}

Kopp, M. U., Crabtree, H. J. Manz, A., 1997, Developments in technology and applications of microsystems, Current Opinion in Chemical Biology, 1, p. 410-419.

Connolly, P., 1995, Clinical diagnostics opportunities for biosensors and bioelectronics, Biosensors \& Bioelectronics, 10, p. 1-6.

Reyes, D. R., Iossifidis, D., Auroux, P., Manz, A., 2002, Micro Total Analysis Systems. 1.Introduction, Theory, and Technology, Anal. Chem., 74, p. 2623-2636.

Manz, A., Graber, N., Widmer, H. M., 1990, Miniaturized total chemical systems: a novel concept for chemical sensing. Sensors and Actuators B, 1, p. 244-248.

Auroux, P., Iossifidis, D., Reyes, D. R., Manz, A., 2002, Micro Total Analysis Systems. 2.Analytical Standard Operations and Applications, Anal. Chem., 74, p. 2637-2652.

Minas, G., Wolffenbuttel, R. F., Correia, J. H., 2005, A Lab-on-a-Chip for Spectrophotometric Analysis of Biological Fluids, Lab-on-a-Chip, 5, p. 1303-1309.

Ribeiro, J. C., Minas, G., Turmezei, P., Wolffenbuttel, R. F., Correia, J. H., 2005, A SU-8 Fluidic Microsystem for Biological Fluids Analysis. Sensors and Actuators A, 123-124 p. 77-81.

Minas, G., Wolffenbuttel, R. F., Correia, J. H., 2006, An array of highly selective Fabry-Perot optical-channels for biological fluids analysis by optical absorption using white light source for illumination. Journal of Optics A: pure and applied optics, 8, p. 272-278.

Minas, G., Martins, J. S., Ribeiro, J. C., Wolffenbuttel, R. F., Correia, J. H., 2004, Biological microsystem for measuring uric acid in biological fluids, Sensors and Actuators A, 110, p. 33-38

Bengtsson, M., Laurell, T., 2004, Ultrasonic agitation in microchannels, Anal Bioanal Chem, 378-7, p. 17161721.

Rife, J.C. et. al., 2000, Miniature valveless ultrasonic pumps and mixers, Sensors and Actuators B, 86, p. $135-140$.

Sessler, G.M, 1987, Topics in Applied Physics: Electrets, 2nd Ed., Springer, Berlin Heidelberg New York.

Sencadas, V., Lanceros-Mendez, S., Mano, J.F., 2004, Thermochimica Acta, 424, p. 201.

Lanceros-Mendez, S., Sencadas, V., Gregorio Filho, R., 2006, Portuguese patent n. ${ }^{\circ} 103318$.

Biochemistry and Organic Reagents: for bioscience investigation. Sigma-Aldrich Diagnostics ${ }^{\circledR}, 2006$.

IBM, Photoresist composition and printed circuit boards and packages made therewith. J. D. Gelorme, R. J. Cox, S. A. R. Gutierrez, US Patent 4882245, 1989.

Candy, J. C., Temes G. C:, 1992, editors, Oversampled Delta-Sigma Data Converters, IEEE Press, New York.

Netravali, A. N., 1997, Optimum digital filters for interpolative A/D converters, Bell Syst. Tech. J., 56, p. 1629. 